\title{
Extração de terceiro molar e suas complicações: revisão de literatura
}

\author{
Third molar extraction and its complications: literature review \\ Extracción del tercer molar y sus complicaciones: revisión de la literatura
}

Recebido: 05/11/2021 | Revisado: 12/11/2021 | Aceito: 16/11/2021 | Publicado: 26/11/2021

\author{
Gabriel Gondim Couto \\ ORCID: https://orcid.org/0000-0002-2216-6744 \\ Faculdade Independente do Nordeste, Brasil \\ E-mail: gabrielcte07@gmail.com \\ Luiz Alfredo Matos Martins \\ ORCID: https://orcid.org/0000-0001-6866-7934 \\ Faculdade Independente do Nordeste, Brasil \\ E-mail: luizalfredo537@gmail.com \\ Milton d' Almeida Ferreira Neto \\ ORCID: https://orcid.org/0000-0002-6895-9133 \\ Faculdade Independente do Nordeste, Brasil \\ E-mail: miltonf_neto@hotmail.com
}

\begin{abstract}
Resumo
A exodontia do terceiro molar é uma prática odontológica com risco potencial de provocar complicações. Diante disso, é necessario que seja realizado um planejamento adequado para prevenir os acidentes transoperatórios e complicações pós-operatórias, visto que embora as cirurgias sejam programadas e executadas por profissionais especializados e capacitados, elas não são isentas de complicações. Foram selecionados 19 estudos indexados nas bases de dados BVS, Lilacsm Scielo e Pubmed. A seleção dos artigos ocorreram de acordo com os critérios de inclusão e exclusão. A leitura na íntegra dos trabalhos fundamentou a escrita da revisão. Diante do exposto, a exérese do terceiro molar implica em complicações como alveolite, hemrragias, lesões nervosas e fraturas. Apesar de bem descritas na literatura, felizmente, elas não são rotineiras, mas existem, Portanto, infere-se a importância do conhecimento e do domínio sobre o tema, tanto para a atuação na prevenção quanto para o manejo frente à situação.
\end{abstract}

Palavras-chave: Complicações pós-operatórias; Extração dentária; Terceiro molar.

\begin{abstract}
Third molar extraction is a dental practice with a potential risk of causing complications. Therefore, it is necessary to carry out an adequate planning to prevent transoperative accidents and postoperative complications, since although surgeries are scheduled and performed by specialized and trained professionals, they are not free from complications. Nineteen studies indexed in the BVS, Lilacs, Scielo and Pubmed databases were selected. The selection of articles took place according to the inclusion and exclusion criteria. The full reading of the papers was the basis for writing the review. Given the above third molar exeresis implies complications such as alveolitis, hemorrhages, nerve injuries and fractures. Although well described in the literature, fortunately, they are not routine, but they do exist. Therefore, it is inferred the importance of knowledge and mastery of the subject, both for acting in prevention and for handling the situation.
\end{abstract}

Keywords: Postoperative complications; Dental extraction; Third molar.

\section{Resumen}

La extracción del tercer molar es una práctica dental con riesgo potencial de causar complicaciones. Por tanto, es necesario realizar una planificación adecuada para prevenir accidentes transoperatorios y complicaciones postoperatorias, ya que si bien las cirugías son programadas y realizadas por profesionales especializados y capacitados, no están libres de complicaciones. Se seleccionaron diecinueve estudios indexados em las bases de datos BVS, Lilacs, Scielo y Pubmed. La selección de artículos se realizó según los criterios de inclusión y exclusión. La lectura completa de los artículos fu ella base para escribir la reseña. Dado lo anterior, la exéresis del tercer molar implica complicaciones como alveolitis, hemorragias, lesiones nerviosas y fracturas. Aunque están bien descritos em la literatura, afortunadamente, no son rutinarios, pero existen. Por tanto, se infiere la importancia del conocimiento y dominio del tema, tanto para actuar em la prevención como para el manejo de la situación.

Palabras clave: Complicaciones posoperatorias; Extracción dental; Tercer molar. 


\section{Introdução}

Os terceiros molares são os últimos dentes na escala cronológica da erupção, e, frequentemente, apresentam-se inclusos ou semi-inclusos. Dados da literatura citam que cerca de $73 \%$ dos jovens da Europa sofrem impactação, seja pela topografia óssea, com a diminuição da maxila, levando à falta de espaço nas arcadas, ou ainda pela dieta moderna que não proporciona um estímulo adequado para o crescimento da mandíbula (Santosh, 2015). De acordo com a American Association of Oral and Maxillofacial Surgeons (AAOMS), a intervenção é realizada conforme as suas indicações, exatamente pelos potenciais riscos de complicações e morbidade que o procedimento envolve (AAOMS, 2016).

Nesse contexto, é essencial que seja realizado um planejamento pré-operatório, para prever o risco cirúrgico e prevenir as intercorrências. Esse plano é iniciado através dos exames clínico, físico e, posteriormente, de imagem. Diante disso, durante a anamnese podemos obter dados específicos da saúde geral do paciente, assim como a história pregressa e a atual (Neto et al., 2017). Alguns dados da própria entrevista já podem ser lembrados para avaliar o risco de complicações. Diversos estudos correlacionam o tabagismo, idade, gênero, uso de contraceptivos orais com a incidência de intercorrências (Santosh, 2015).

Após a realização do exame clínico e físico é observada a análise radiográfica ou tomográfica para a escolha da técnica cirúrgica, (Machado, 2020) a radiografia panorâmica (PAN) é o exame de imagem padrão para o planejamento préoperatório (Vieira et al., 2020) visto que é um método útil para avaliar o grau de dificuldade cirúrgica, a morfologia, posição, proximidade de estruturas, sendo amplamente disponível e de baixo custo (Gu et al., 2018). Contudo, a PAN pode apresentar algumas distorções geométricas devido a sua avaliação bidimensional ( $\mathrm{Gu}$ et al., 2018) e, por isso, para se determinar a relação entre as estruturas e formas anatômicas o seu diagnóstico é pobre (Vieira et al., 2020).

Além da realização de todo o plano cirúrgico, avaliação das estruturas e escolha da técnica adequada, outras medidas devem ser tomadas para reduzir as chances de complicações pós-operatórias. Dentre elas, a biossegurança adequada: materiais e campo cirúrgico estéril, além da realização de antissepsia extra-oral e intra-oral. Na sequência, a escolha correta da técnica anestésica, do sal anestésico e do retalho cirúrgico, somados com a destreza e habilidade do cirurgião (Ferreira \& Mandarino, 2019).

Diante da temática exposta, é necessário que seja realizado um planejamento adequado para prevenir os acidentes no transoperatórios e complicações pós-operatórias, visto que, embora as cirurgias sejam programadas e executadas por profissionais especializados e capacitados, elas não estão isentas de complicações (Costa et al., 2020). Dessa forma, de acordo com a alta prevalência na realização da extração do terceiro molar, enaltece-se que é de extrema relevância realizar uma revisão de literatura narrativa acerca do tema, abordando as principais complicações relacionadas ao procedimento, a fim de preparar o profissional para intervir de forma resolutiva frente aos possíveis eventos que possam vir a ocorrer.

\section{Metodologia}

Segundo Severino (2013), o trabalho científico precisa recorrer de um volume de fontes para o desenvolvimento do raciocínio demonstrativo. A heurística é a ciência, técnica e arte de localização e levantamento de documentos. Sendo assim, é constituída de uma série de procedimentos para a busca metódica e sistemática dos documentos que possam interessar ao tema que se pesquisa. Este estudo trata-se de uma revisão de literatura descritiva, fundamentada através de dados selecionados de fontes secundárias como trabalhos acadêmicos, artigos, livros de referência, jornais e revistas. Onde foram consultadas as base de dados BVS (Biblioteca Virtual em Saúde), LILACS (Literatura Latino-Americana e do Caribe em Ciências da Saúde), SciELO (Scientific Eletronic Library onLine) e PubMed, fazendo-se um levantamento qualitativo de artigos, utilizando o operador booleano "and" com os seguintes descritores: Complicações (Complications), Terceiro molar (Third Molar), Indicações (Indications), Pós-operatório (Postoperative). 
Os critérios utilizados para a seleção foram: artigos que descreveram as complicações da extração do terceiro molar, estudos que relataram a utilização de exames de imagem para o planejamento cirúrgico, publicados no período de 2013 a 2021 na língua inglesa e portuguesa. Foram excluídos artigos sem correlação entre as complicações e o procedimento de extração do terceiro molar e estudos em outras línguas. A realização da operacionalização da revisão deu-se na seguinte ordem: seleção do descritor, busca dos artigos nas bases de dados citadas, leitura do título, resumo e conclusão, aplicação dos critérios de inclusão ou exclusão para a seleção dos estudos, leitura completa dos artigos selecionados e elaboração do trabalho.

\section{Resultados}

Nas buscas realizadas nas bases de dados: LILACS, BVS, Pubmed e Scielo, com o auxílio dos descritores mencionados foram obtidos como resultado, respectivamente: 221, 836, 330 e 512 artigos. Após a leitura do título e resumo dos resultados da pesquisa e aplicação dos critérios de inclusão e exclusão, dos 221 artigos encontrados no LILACS nenhum deles foi selecionado, pois não se enquadravam no projeto, dos 836 artigos da BVS apenas seis foram selecionados (Matzen \& Wenzel, 2015; Matzen \& Berkhout, 2019; Rodrigues, 2014; Costa et al., 2020; Gopee \& Rikhotso, 2017; Sreesha et al., 2020).

No Pubmed foi utilizado o recurso MeSH Terms, dos 330 artigos encontrados, oito foram inseridos na revisão (Klinder et al., 2019; Santosh, 2015; Ryalat et al., 2017; Sayed et al., 2018; Leung, 2019; Gu et al., 2018; Seguro \& Oliveira, 2014; Filho et al., 2020). Na base de dado Scielo foram encontrados 512 artigos na busca, mas apenas cinco foram incluídos no trabalho (Ferreira \& Mandarino, 2019; Neto et al., 2017; Machado, 2020; Vieira et al., 2020; Cho et al., 2017).

Após a seleção e a leitura íntegra dos artigos mencionados foi dada a elaboração do trabalho. Os resultados dos artigos incluídos nesta revisão estão expostos no quadro a seguir.

Quadro 1. Resultados do levantamento bibliográfico.

\begin{tabular}{|c|c|c|}
\hline Autor /ano & Título do trabalho & Resultados \\
\hline Rodrigues, 2014. & $\begin{array}{l}\text { Avaliação da precisão da Radiografia } \\
\text { Panorâmica no planejamento cirúrgico } \\
\text { para extração dos terceiros molares } \\
\text { inferiores. }\end{array}$ & $\begin{array}{l}\text { A radiografia panorâmica foi uma ferramenta útil e confiável no planejamento pré- } \\
\text { operatório para os casos de extração de dentes terceiros molares inferiores. Nos casos } \\
\text { atendidos, não ocorreram acidentes ou complicações relacionados a um planejamento } \\
\text { radiográfico pré-operatório deficiente. }\end{array}$ \\
\hline $\begin{array}{l}\text { Seguro; oliveira, } \\
2014 .\end{array}$ & $\begin{array}{l}\text { Complicações Pós-Cirúrgicas na remoção } \\
\text { de terceiros molares inclusos. }\end{array}$ & $\begin{array}{l}\text { As complicações obtidas foram trismo, comunicação buco-sinusal, infecção local, } \\
\text { alveolite, dor pós-operatória, edema, fratura da tuberosidade da maxila e fratura de } \\
\text { mandíbula. }\end{array}$ \\
\hline $\begin{array}{l}\text { Matzen; wenzel, } \\
2015 .\end{array}$ & $\begin{array}{l}\text { Efficacy of CBCT for assessment of } \\
\text { impacted mandibular third molars: a } \\
\text { review - based on a hierarchical model of } \\
\text { evidence }\end{array}$ & $\begin{array}{l}\text { A radiografia pode ser suficiente na maioria dos casos antes da remoção do terceiro molar } \\
\text { mandibular, mas a Tomografia Computadoriza de Feixe Cônico pode ser sugerida quando } \\
\text { um ou mais sinais para um contato próximo entre o dente e o canal mandibular estão } \\
\text { presentes. Se acredita que a CBCT mudará o tratamento ou o resultado, diminuindo as } \\
\text { complicações. }\end{array}$ \\
\hline Santosh, 2015. & $\begin{array}{l}\text { Impacted Mandibular Third Molar: } \\
\text { Review of literature and a proposal of a } \\
\text { combined clinical and radiological } \\
\text { classification. }\end{array}$ & $\begin{array}{l}\text { As complicações mais comuns associadas à remoção do terceiro molar incluem dor, lesão } \\
\text { do nervo }(0,5 \% \text { a } 20 \%) \text {, alvéolo seco }(0 \text { a } 35 \%) \text {, infecção e hemorragia. Trismo grave, } \\
\text { fístula oroantral, hérnias de gordura bucal, dano iatrogênico ao segundo molar adjacente e } \\
\text { fratura mandibular iatrogênica também podem ocorrer, embora muito raramente. }\end{array}$ \\
\hline Cho et al., 2017. & $\begin{array}{l}\text { Postoperative interventions to reduce } \\
\text { inflammatory complications after molar } \\
\text { surgery: review of the current evidence. }\end{array}$ & $\begin{array}{l}\text { Complicações inflamatórias como dor, edema, trismo, infecção e osteíte alveolar têm } \\
\text { efeito adverso na qualidade de vida dos pacientes após a remoção do terceiro molar. Esta } \\
\text { revisão apresenta as evidências atuais sobre estratégias pós-operatórias para reduzir essas } \\
\text { complicações (anti-inflamatórios, corticoides, crioterapia e irrigação). }\end{array}$ \\
\hline $\begin{array}{l}\text { Gopee; rikhotso, } \\
2017 .\end{array}$ & $\begin{array}{l}\text { Impacted mandibular third molars: the } \\
\text { efficacy of prophylactic antibiotics and } \\
\text { chlorhexidine mouthwash in preventing } \\
\text { postoperative infections. }\end{array}$ & $\begin{array}{l}\text { A taxa de infecção foi de } 8 \% \text { e } 6 \% \text { para os grupos. Nenhuma diferença estatisticamente } \\
\text { significativa na cirurgia foi encontrada entre os dois grupos (uso de antibióticos e limpeza } \\
\text { com clorexidina e enxaguantes bucais). }\end{array}$ \\
\hline Neto et al., 2017. & $\begin{array}{l}\text { Principais Complicações das Cirurgias } \\
\text { de terceiros molares: revisão de literatura. }\end{array}$ & Hemorragias, fraturas mandibulares, lesão do nervo lingual e do nervo alveolar inferior. \\
\hline Gu et al., 2018. & $\begin{array}{l}\text { Anatomic study of the position of the } \\
\text { mandibular canal and corresponding } \\
\text { mandibular third molar on cone-beam } \\
\text { computed tomography images. }\end{array}$ & $\begin{array}{l}\text { As posições horizontal e vertical do canal mandibular e os terceiros molares } \\
\text { correspondentes são um fator anatômico chave da lesão do nervo alveolar inferior. }\end{array}$ \\
\hline $\begin{array}{l}\text { Ryalat et al., } \\
2018 .\end{array}$ & $\begin{array}{l}\text { Impaction of lower third molars and their } \\
\text { association with age: radiological } \\
\text { perspectives. }\end{array}$ & $\begin{array}{l}\text { As complicações mais esperadas após a cirurgia do terceiro molar incluem lesão do nervo } \\
\text { sensorial, alvéolo seco, dor, inchaço, trismo, infecção e hemorragia. Essas complicações } \\
\text { são perturbadoras para pacientes jovens, especialmente se eles se tornarem permanentes } \\
\text { em casos de lesões do nervo alveolar inferior ou lingual. Além de fatores relacionados à } \\
\text { cirurgia, a posição e angulações de terceiros molares estão fortemente associadas com o }\end{array}$ \\
\hline
\end{tabular}




\begin{tabular}{|c|c|c|}
\hline & & número e o grau de morbidades pós-operatórias. \\
\hline $\begin{array}{c}\text { Ferreira; } \\
\text { mandarino, } 2019 .\end{array}$ & $\begin{array}{l}\text { Complications obtained at the post- } \\
\text { operative exodontia of third-party molars. }\end{array}$ & $\begin{array}{l}\text { O escopo deste estudo foi avaliar a incidência de acidentes e complicações relacionadas à } \\
\text { exodontia de terceiros molares. Dentre as principais intercorrências tivemos, edema } \\
(29 \%) \text {, trismo }(26 \%) \text {, seguida pela dor }(18 \%) \text {, hemorragia }(18 \%) \text { e parestesia }(9 \%) \text {. A } \\
\text { condição de alveolite não foi identificada nos sinais clínicos analisados nos pacientes } \\
(0 \%) \text {. }\end{array}$ \\
\hline $\begin{array}{l}\text { Klinder et al., } \\
\quad 2019 .\end{array}$ & $\begin{array}{l}\text { Does craniofacial morphology affect third } \\
\text { molars impaction? Results from a } \\
\text { population-based in northeastern } \\
\text { Germany. }\end{array}$ & $\begin{array}{l}\text { Indivíduos com largura craniana máxima, face anterior total ou antero-inferior e índice } \\
\text { facial inferior têm um risco aumentado de impactação de terceiros molares mandibulares. } \\
\text { Esses achados podem ajudar dentistas ortodônticos, cirurgiões orais e maxilofaciais na } \\
\text { tomada de decisão para remoção de terceiros molares em seu tratamento. Não houve } \\
\text { relação com risco de complicações no estudo. }\end{array}$ \\
\hline Leung, 2019. & $\begin{array}{l}\text { Management and prevention of third molar } \\
\text { surgery-related trigeminal nerve injury: } \\
\text { time for rethink. }\end{array}$ & $\begin{array}{l}\text { A lesão do nervo trigêmeo como consequência da cirurgia do terceiro molar inferior é } \\
\text { uma complicação notória e pode afetar o paciente em longo prazo. A lesão do nervo } \\
\text { alveolar inferior (IAN) e do nervo lingual (LN) resulta em diferentes graus de déficit } \\
\text { neurossensorial e também em outros sintomas neurológicos. }\end{array}$ \\
\hline $\begin{array}{c}\text { Matzen; } \\
\text { berkhout, } 2019 .\end{array}$ & $\begin{array}{l}\text { Cone beam CT imaging of the mandibular } \\
\text { third molar: a position paper prepared by } \\
\text { the European Academy of } \\
\text { DentoMaxilloFacialRadiology } \\
\text { (EADMFR) }\end{array}$ & $\begin{array}{l}\text { Recomendações baseadas em evidências defendem que a Tomografia para avaliar o } \\
\text { terceiro molar não deve ser aplicada como método de rotina antes da sua remoção, apenas } \\
\text { em casos onde o cirurgião tem uma questão clínica muito específica que não pode ser } \\
\text { respondida pela imagem convencional (panorâmica). }\end{array}$ \\
\hline $\begin{array}{l}\text { Sayed et al., } \\
\quad 2019 .\end{array}$ & $\begin{array}{l}\text { Complications of Third Molar Extraction: } \\
\text { a retrospective study from a tertiary } \\
\text { healthcare centre in Oman. }\end{array}$ & $\begin{array}{l}\text { Entre os } 625 \text { terceiros molares inferiores extraídos, } 45 \text { casos relataram lesões nervosas, } \\
\text { das quais a maioria } \\
(91,1 \%) \text { eram lesões temporárias }(\mathrm{LN}=71,1 \% \text {; IAN }=20 \%) \text {, e poucos }(8,9 \%) \text { eram } \\
\text { lesões permanentes }(\mathrm{LN}=6,7 \% \text {; IAN }=2,2 \%) \text {. Com base no total extraído mandibular } \\
\text { terceiros molares, a taxa geral de danos permanentes aos nervos foi encontrada para ser } \\
0,7 \% \text { (LN }=0,5 \% ; \text { IAN }=0,2 \%) \text {, enquanto a lesão nervosa temporária geral foi de } 6,5 \% \\
(\mathrm{LN}=5,1 \% \text {; IAN }=1,4 \%) \text {. }\end{array}$ \\
\hline Costa et al., 2020. & $\begin{array}{l}\text { Complicações e intercorrências associadas } \\
\text { a exodontia de terceiros molares na clínica } \\
\text { de odontologia da faculdade vértice- } \\
\text { univertix }\end{array}$ & $\begin{array}{l}\text { A dor e o edema pós-operatório podem ocorrer nas primeiras } 24 \text { horas, sendo o pico } \\
\text { máximo do edema dentro de } 48 \text { a } 72 \text { horas. Trismo, Hemorragias, Alveolite e danos em } \\
\text { estruturas nervosas podem ocorrer. }\end{array}$ \\
\hline Filho et al., 2020. & $\begin{array}{l}\text { Acidentes e complicações associados a } \\
\text { exodontia de terceiros molares -Revisão da } \\
\text { literatura. }\end{array}$ & $\begin{array}{l}\text { Entre as complicações pós-operatórias mais comuns inerentes a exodontia de elementos } \\
\text { dentários, está a alveolite. A ocorrência dessas intercorrências é mais evidente quando } \\
\text { realizadas por profissionais recém-formados e/ou inexperientes, pois, o cirurgião dentista } \\
\text { ou cirurgião bucomaxilofacial tem que estar totalmente preparado para realizar o } \\
\text { procedimento. A falta de conhecimento anatômico e das técnicas utilizadas podem gerar } \\
\text { acidentes durante o transoperatório e complicações ainda maiores no pós operatório }\end{array}$ \\
\hline Machado, 2020. & $\begin{array}{l}\text { Acidentes e complicações associados a } \\
\text { extração de terceiro molar. }\end{array}$ & $\begin{array}{l}\text { Complicações trans operatórias: fraturas dentoalveolares, danos à dentes adjacentes e/ou } \\
\text { ATM, fratura de mandíbula, comunicação bucosinusal, deslocamento de dentes para } \\
\text { regiões anatômicas nobres. Complicações pós-operatórias: dor, trismo, hemorragia, } \\
\text { edema, lesão do nervo alveolar inferior e alveolite. }\end{array}$ \\
\hline $\begin{array}{l}\text { Sreesha et al., } \\
2020 .\end{array}$ & $\begin{array}{l}\text { Posoperative pain, edema and trismus } \\
\text { following third molar surgery - A } \\
\text { comparative study between submucosal } \\
\text { and intravenous dexamethasone. }\end{array}$ & $\begin{array}{l}\text { A remoção cirúrgica do terceiro molar inferior costuma estar associado a desconforto } \\
\text { pós-operatório, como dor, inchaço e trismo. A gravidade dessas sequelas pós-operatórias } \\
\text { depende do manuseio dos tecidos moles durante o período intraoperatório, da extensão da } \\
\text { osteotomia e da duração do procedimento cirúrgicos. Foi realizada a administração da } \\
\text { dexametasona venosa e submucosa para o controle dos efeitos pós-operatórios, sendo } \\
\text { observada que a administração da dexametasona por via submucosa é benéfica para a } \\
\text { adesão geral do paciente. }\end{array}$ \\
\hline $\begin{array}{l}\text { Vieira et al., } \\
2020 .\end{array}$ & $\begin{array}{l}\text { Influência de diferentes exames por } \\
\text { imagem no planejamento cirúrgico de } \\
\text { terceiros molares inferiores: uma revisão } \\
\text { de literatura. }\end{array}$ & $\begin{array}{l}\text { Vários são os riscos e complicações dos procedimentos cirúrgicos relatados incluindo } \\
\text { sangramento, infecção, fratura do maxilar, danos dos nervos lingual e nervo alveolar } \\
\text { inferior. }\end{array}$ \\
\hline
\end{tabular}

Fonte: Autores (2021).

\section{Discussão}

O pós-operatório da extração do terceiro molar está relacionado à resposta inflamatória ao ato cirúrgico: dor, edema, trismo, são os sintomas mais prevalentes e descritos na literatura. Nesse contexto, o uso de anti-inflamatórios, enxaguantes bucais e corticoides podem ser indicados de acordo com a avaliação clínica do Cirurgião Dentista (Costa et al., 2020; Gopee \& Rikhotso, 2017; Machado, 2020; Sreesha et al., 2020). Entretanto, complicações de cunho infeccioso, lesões nervosas, hemorragias e fraturas também podem ocorrer e acabam tendo uma repercussão negativa na qualidade de vida do paciente (Gopee \& Rikhotso, 2017; Neto et al., 2017; Cho et al., 2017; Costa et al., 2020).

O quadro infeccioso inclui osteíte alveolar, osteomielite, linfadenopatia (localizada/generalizada) e envolvimento do espaço fascial. Nesses casos, a antibioticoterapia se faz necessária. Todavia, de acordo com Goope \& Rikhotso (2017) e Costa et. al. (2020) a antibioticoprofilaxia de rotina não é recomendada, pois já é bem descrito na literatura que a prevenção com amoxicilina e clorexidina são igualmente eficazes na redução das infecções pós-operatórias em cirurgias de terceiro molar 
(Gopee \& Rikhotso, 2017; Costa et al., 2020). No entanto, diversas complicações não infecciosas são susceptíveis na abordagem cirúrgica da extração do terceiro molar.

A disfunção nervosa é uma delas, seja ela do nervo lingual, trigêmeo, alveolar inferior, provocando sintomas como parestesia e déficits neurossensoriais transitórios ou permanentes (Gu et al., 2018; Sayed et al., 2019; Leung, 2019; Costa et al., 2020). Diante disso, a avaliação pré-operatória com exames de imagem mais acurados seja ela a Radiografia Panorâmica ou a Tomografia Computadorizada de Feixe Cônico devem ser realizados para avaliar o risco de lesão antes do procedimento cirúrgico. Segundo Klinder et al. (2019), a posição horizontal e vertical do canal mandibular e terço correspondente aos molares mostram estreita relação e um maior risco (Klinder et al., 2019). No entanto, novas tecnologias, como as funções da eritropoietina e das terapias com células-tronco estão sendo investigadas para a neuroproteção e regeneração neural, apesar de serem terapias promissoras, a prevenção das lesões neuronais continua sendo a primeira escolha (Gu et al., 2018; Sayed et al., 2019; Leung, 2019; Costa et al., 2020; Machado, 2020).

A hemorragia, outra complicação possível, é caracterizada pelo extravasamento maciço de sangue, nesses casos não ocorre o mecanismo adequado da hemostasia, prejudicando assim, a coagulação sanguínea, fator responsável para a contenção do sangramento. As hemorragias podem ocorrer durante ou após a extração, vale ressaltar, que o ambiente oral é amplamente vascularizado e a probabilidade de ressecção de algum vaso sanguíneo deve ser considerada (Costa et al., 2020; Neto et al., 2017; Filho et al., 2020).

A fratura, bem menos recorrente, ocorre quando a força do osso é superada pelas forças que atuam sobre ele. Inúmeros fatores afetam na incidência dessa complicação, sendo eles o grau de impactação dos dentes, o tipo de angulação, o comprimento das raízes, idade do paciente, a experiência do cirurgião, a presença de neoplasias em torno do terceiro molar, o uso de medicamentos que prejudicam a qualidade dos ossos, diabetes, fatores fisiológicos ligados ao metabolismo do cálcio e um exame pré-operatório inadequado (Neto et al., 2017; Machado, 2020).

A fratura de mandíbula e a da tuberosidade da maxila, são complicações raras, de acordo com Seguro \& Oliveira (2014) as fraturas peri-operatórias são resultado do uso incorreto da alavanca associado ao desgaste excessivo do osso (Seguro \& Oliveira, 2014), já as fraturas no pós-operatórios estão relacionadas com o resultado do elevado nível de força na mordida durante a mastigação, podendo ocorrer entre a segunda e terceira semana, por isso, a primeira semana é considerada o período crítico para a ocorrência de fraturas, sendo necessária a manutenção da dieta líquido-pastosa (Neto et al., 2017; Machado, 2020).

Diante do exposto, as complicações relacionadas à extração do terceiro molar podem variar desde simples quadros inflamatórios até mesmo disfunções permanentes e pior prognóstico. Por isso, a avaliação clínica dispondo-se de métodos mais abrangentes, auxilia no diagnóstico pré-operatório para avaliar com maestria os riscos da extração do terceiro molar. A radiografia panorâmica é realizada rotineiramente antes da sua extração e na grande maioria dos casos, a imagem panorâmica se faz suficiente. Contudo, em casos mais complexos, a avaliação pela Tomografia Computadorizada de Feixe Cônico pode fornecer informações adicionais. O pressuposto subjacente é a visualização mais acurada que pode levar a uma intervenção cirúrgica com visão guiada, diminuindo consideravelmente o risco de induzir danos (Matzen \& Wenzel, 2015; Matzen \& Berkhout, 2019; Rodrigues, 2014; Ryalat et al., 2018; Vieira et al., 2020).

Sendo assim, nesse contexto, cabe ao profissional esclarecer previamente e conscientizar o paciente sobre os possíveis acometimentos, além de realizar os cuidados pré, trans e pós-operatórios para prever o risco e conduzir o procedimento. A avaliação clínica minuciosa com a história e o exame físico completos associados à solicitação de exames complementares (Radiografia ou Tomografia) são imprescindíveis, assim como, a qualificação e experiência do Cirurgião-Dentista (Machado, 2020; Neto et al., 2017). 


\section{Conclusão}

O risco de intercorrências ou complicações são passíveis em qualquer procedimento invasivo, principalmente, em procedimentos cirúrgicos. Diversos fatores influenciam para uma elevada probabilidade desses acontecimentos, sejam eles, fatores relacionados ao paciente (idade, sexo, comorbidades, angulação do terceiro molar e a relação com as estruturas adjacentes) ou fatores relacionados ao próprio ato (duração, assepsia adequada, técnica cirúrgica). Dessa forma, conclui-se que a exérese do terceiro molar também implica em diversas complicações, sendo elas as mais descritas na literatura: alveolite, hemorragias, lesões nervosas e fraturas. Felizmente, essas intercorrências não são rotineiras, mas também não são inexistentes. Diante disso, é necessário que os próximos estudos levem em consideração os fatores intrínsecos dos pacientes associados com as questões técnicas do procedimento e da avaliação pré-operatória, afim de se obter desfechos clínicos conclusivos para a redução das referidas complicações. Por isso, infere-se a importância do conhecimento sobre as possíveis complicações, prevenção quanto ao risco e o manejo adequado frente à situação.

\section{Referências}

American Association of Oral and Maxillofacial Surgeons. (2016). Management of Third Molar Teeth. https://www.aao ms.org/docs/govt_affairs/advocacy_white_papers/management_third_molar_white_paper.pdf

Costa, H. C., Póvoa, I. H. X., Barros, W. L., Queiroz, G. E. R., Avelar, J. C. (2020). Complicações e intercorrências associadas a exodontia de terceiros molares na clínica de odontologia da faculdade vértice-univertix, Anais XIII FAVE. Recuperado de https://fave.univertix.net/wp-content/uploads/2020/11/A-3COMPLICACOES-E-INTERCORRENCIAS-ASSOCIADAS-A-EXODONTIA-DE-TERCEIROS-MOLARES.pdf

Cho, H., Lynham, A. J., Hsu, E. (2017). Postoperative interventions to reduce inflammatory complications after molar surgery: review of the current evidence. Review Aust Dent J 62 (4), 412-419. Recuperado de https://onlinelibrary.wiley.com/doi/10.1111/adj.12526

Ferreira, A. C. P., Mandarino, S. C. A. (2019). Complications obtained at the post-operative exodontia of third-party molars. Cadernos de Odontologia do UNIFESO, 1 (1), 26-36. Recuperado de http://www.revista.unifeso.edu.br/index.php/cadernosodontologiaunifeso/article/view/1774/707

Filho, M. J. S. F., Silva, H. R. F., Rosário, M. S. R., Takano, V. Y. S., Nascimento, J. R., Aguiar, J. L., Milério, L. R., Pimenta, Y. S. (2020). Acidentes e complicações associadas a exodontia de terceiros molares - Revisão de Literatura. Braz. J. of Develop. 6 (11), 93650-65. https://www.brazilianjournals.com/index.php/BRJD/article/view/20781/16602

Gopee, P., Rikhotso, E. (2017). Impacted mandibular third molars: the efficacy of prophylactic antibiotics and chlorhexidine mouthwash in preventing postoperative infections. S. Afr. Dente J. 72 (5), 213-218. http://www.scielo.org.za/pdf/sadj/v72n5/04.pdf

Gu, L., Zhu, C., Chen. K., Liu, X., Tang, Z. (2018). Anatomic study of the position of the mandibular canal and corresponding mandibular third molar on cone-beam computed tomography image. Surg Radiol Anat, 40 (6), 609-614. https://www.ncbi.nlm.nih.gov/pmc/articles/PMC5958164/

Kindler, S., Ittermann, T., Bülow, R., Holtfreter, B., Klausenitz, C., Metelmann, P., Mksoud, M., Pink, C., Seebauer, C., Kocher, T., Koppe, T., Krey, K. F., Metelmann, H. R., Völzke, H., Daboul, A. (2019). Does craniofacial morphology affect third molars impaction? Results from a population-based in northeastern Germany. PLoS One 14 (11), 1-16. Recuperado de https://www.ncbi.nlm.nih.gov/pmc/articles/PMC6874347/

Leung, Y. (2019). Management and prevention of third molar surgery-related trigeminal nerve injury: time for rethink. J Korean Assoc Oral Maxillofac Surg, 45 (5), 233-240. https://www.ncbi.nlm.nih.gov/pmc/articles/PMC6838349/

Machado, W. M. (2020). Acidentes e complicações associados a extração de terceiro molar. Trabalho de conclusão de curso [Título de cirurgião dentista] Centro Universitário Uniguairacá de Guarapuava. http://200.150.122.211:8080/jspui/bitstream/23102004/251/1/Acidentes\%20e\%20compl ica\%c3\%a7\%c3\%b5es\%20associados\%20a\%20extra\%c3\%a7\%c3\%a3o\%20de\%20terceiro\%20molar.pdf

Matzen, L. H., Berkhout, E. (2019). Cone beam CT imaging $\mathrm{f}$ the mandibular third molar: a posicion paper prepared by the European Academy of DentoMaxilloFacial Radiology (EADMFR). Dentomaxillofac Radiol 48 (5), 1-5. https://www.birpublications.org/doi/pdf/10.1259/dmfr.20190039

Matzen, L. H., Wenzel, A. (2015). Efficacy of CBCT for assessment of impacted mandibular third molars: a review - based on a hierarchical model of evidence. Dentomaxillofac Radiol 44 (1), 1-11. https://www.ncbi.nlm.nih.gov/pmc/articles/PMC4277437/

Neto, O. B., Igarçaba, M., Fernandes, B. R., Pereira, R., Ribeiro, J., Vieira, E. H. (2017). Principais complicações das cirurgias de terceiros molares: revisão de literatura, Rev Ciência Atual, 10 (2), 1-8. http://www.cnad.edu.br/revista-ciencia-atual/index.php/cafsj/article/view/201/pdf.

Ryalat, S., Alryalat, S. A., Kassob, Z., Hassona, Y., Al-Shayyab, M. H. (2018). Impaction of lower third molars and their association with age: radiological perspectives. BMC Oral Health 18 (1), 1-5. https://www.ncbi.nlm.nih.gov/pmc/articles/PMC5885350/

Rodrigues, V. P. (2014). Avaliação da precisão da radiografia panorâmica no planejamento cirúrgico para extração de terceiros molares inferiores. Brasília. Trabalho de conclusão de curso [Graduação em odontologia] - Departamento de Odontologia da Faculdade de Ciências da Saúde da Universidade de Brasília. https://bdm.unb.br/bitstream/10483/9494/1/2014_WanessaPortilhoRodrigues.pdf 
Research, Society and Development, v. 10, n. 15, e268101522873, 2021

(CC BY 4.0) | ISSN 2525-3409 | DOI: http://dx.doi.org/10.33448/rsd-v10i15.22873

Sayed, N., Bakathir, A., Pasha, M., Al-Sudairy, S. (2019). Complications of Third Molar Extraction: a retrospective study from a tertiary healthcare centre in Oman. Clinical and Basic Research, 19 (5), 230-235. https://www.ncbi.nlm.nih.gov/pmc/articles/PMC6839670/

Santosh, P. (2015). Impacted Mandibular Third Molar: Review of literature and a proposal of a combined clinical and radiological classification, Ann Med Health Sci Res, 5 (4), 229-234. https://www.ncbi.nlm.nih.gov/pmc/articles/PMC4512113/

Seguro, D., Oliveira, R. V. (2014). Complicações pós-cirúrgicas na remoção de terceiros molares inclusos. Revista UNING 20 (1), $30-34$. http://revista.uninga.br/index.php/uningareviews/article/view/1572

Severino, A. J. (2013). Metodologia do trabalho científico [livro eletrônico], Cortez, https://www.ufrb.edu.br/ccaab/images/AEPE/Divulga\%C3\%A 7\%C3\%A3o/LIVROS/Metodologia_do_Trabalho_Cient\%C3\%ADfico_-_1\%C2\%AA_Edi\%C3\%A7\%C3\%A3o_-_Antonio_Joaquim_Severino_-_2014.pdf

Sreesha, A., Ummar, M., Sooraj, S., Aslam, S., Roshni, A., Jabir, K. (2020). Posoperative pain, edema and trismus following third molar surgery - A comparative study between submucosal and intravenous dexamethasone. J Family Med Prim Care 9 (5), 2454-2459. https://www.ncbi.nlm.nih.gov/pmc/articles/PMC7380777/

Vieira, A. L., Rosado, L. P. L., Oliveira, M. L. B., Nunes, W. J. P., Junqueira, R. B., Castro, M. A. A., Carvalho, M. F., Verner, F. S. (2020). Influência de diferentes exames por imagem no planejamento cirúrgico de terceiros molares inferiores: uma revisão de literatura. HU Rev, 45 (1), 13-21. https://periodicos.ufjf.br/index.php/hurevista/article/view/29530/20966 